\title{
Characterizations of slant and spherical helices due to pseudo-Sabban frame
}

\author{
Bülent Altunkaya $^{\mathrm{a}^{*}}$ and Levent Kula ${ }^{\mathrm{a}}$ \\ ${ }^{a}$ Department of Mathematics, Faculty of Education, University of Ahi Evran, Kirşehir, Turkey \\ *Corresponding author E-mail: bulent.altunkaya@ahievran.edu.tr
}

\section{Article Info}

Keywords: Geodesic curvature, Pseudosphere, Hyperbolic plane, Helix, Sabban frame, Minkowski space

2010 AMS: 51B20, 53A04

Received: 3 April 2018

Accepted: 19 April 2018

Available online: 30 June 2018

\begin{abstract}
In this paper, we investigate that under which conditions of the geodesic curvature of unit speed curve $\gamma$ that lies on $S_{1}^{2}$ or $H^{2}$, the curve $\alpha$ which is obtained by using $\gamma$, is a spherical helix or slant helix in Minkowski 3-space.
\end{abstract}

\section{Introduction}

There are several studies in literature examining methodology to use spherical curves to construct some specialized curves. For example, Izuyama and Takeuchi [7], defined a way to construct Bertrand curves from the spherical curve whose spherical evolute coincides with the spherical Darboux image of the Bertrand curve. In addition to this paper, Encheva and Georgiev [4] showed a way to construct all Frenet curves $(\kappa>0)$ by the following formula

$$
\alpha(s)=b \int e^{\int k(s) d s} \gamma(s) d s+a
$$

where $b$ is a constant number, $a$ is a constant vector, $\gamma$ is a unit speed curve on $S^{2}$ with the Sabban frame and $k: I \rightarrow R$ is a function of class $C^{1}$. Moreover, they showed that the spherical curve $\gamma$ is a circle if and only if the corresponding Frenet curves are cylindrical helices. Previously, we have found some characterizations to construct spherical helices and slant helices in Euclidean space by using these methods [2].

This paper is organized in the following way. In section 2 basic concepts of Minkowski 3-space $R_{1}^{3}$ are given. In section 3, spherical helices in $R_{1}^{3}$ are discussed by indicating some examples. Similarly, in section 4 , slant helices in $R_{1}^{3}$ are examined.

\section{Basic Concepts}

Let us consider the Minkowski 3-space $R_{1}^{3}$ with the Lorentzian inner product

$$
\langle x, y\rangle=x_{1} y_{1}+x_{2} y_{2}-x_{3} y_{3}
$$

where $x=\left(x_{1}, x_{2}, x_{3}\right)$ and $y=\left(y_{1}, y_{2}, y_{3}\right) \in R_{1}{ }^{3}$. The pseudo-norm of a vector $x$ is given by $\|x\|=\sqrt{|\langle x, x\rangle|}$. In the space $R_{1}^{3}$, the Lorentzian cross-product is defined as follows

$$
x \wedge y=\left|\begin{array}{ccc}
e_{1} & e_{2} & -e_{3} \\
x_{1} & x_{2} & x_{3} \\
y_{1} & y_{2} & y_{3}
\end{array}\right|=\left(\begin{array}{lll}
\left.x_{2} y_{3}-x_{3} y_{2}, \quad x_{3} y_{1}-x_{1} y_{3}, \quad x_{2} y_{1}-x_{1} y_{2}\right)
\end{array}\right.
$$


It's clearly seen that the cross-product has the following properties [3],

$$
\begin{aligned}
& \text { (i) } \quad x \wedge y=-(y \wedge x) \\
& \text { (ii) } \quad\langle x \wedge y, z\rangle=\operatorname{det}(x, y, z) \\
& \text { (iii) } \quad x \wedge(y \wedge z)=\langle x, y\rangle z-\langle x, z\rangle y \\
& \text { (iv) } \quad\langle x \wedge y, x \wedge y\rangle=(\langle x, y\rangle)^{2}-\langle x, x\rangle\langle y, y\rangle \\
& \text { (v) } \quad\langle x \wedge y, x\rangle=0 \quad, \quad\langle x \wedge y, y\rangle=0
\end{aligned}
$$

where $x, y, z \in R_{1}^{3}$.

A vector $x \in R_{1}{ }^{3}$ is called spacelike if $\langle x, x\rangle>0$ or $x=0$, timelike if $\langle x, x\rangle<0$, lightlike if $\langle x, x\rangle=0$ and $x \neq 0$ [8].

In [8], the hyperbolic plane (resp. pseudosphere) center $q \in R_{1}^{3}$ and of radius $r>0$ are defined by,

$$
\begin{gathered}
H^{2}(r ; q)=\left\{x=\left(x_{1}, x_{2}, x_{3}\right) \in R_{1}^{3}:\langle x-q, x-q\rangle=-r^{2}, x_{3}-q_{3}>0\right\}, \\
S_{1}^{2}(r ; q)=\left\{\left(x_{1}, x_{2}, x_{3}\right) \in R_{1}^{3}:\langle x-q, x-q\rangle=r^{2}\right\} .
\end{gathered}
$$

When $r=1$ and $p$ is the origin, the hyperbolic plane is denoted by $H^{2}$ and the pseudosphere is denoted by $S_{1}^{2}$. In this paper, when a helix lies on $H^{2}(r ; q)$ or $S_{1}^{2}(r ; q)$, we call it spherical curve.

Given a regular curve $\alpha(t): I \subset R \rightarrow R_{1}^{3}$. We say that $\alpha$ is spacelike (resp. timelike, lightlike) at $t$ if $\alpha^{\prime}(t)$ is a spacelike (resp. timelike, lightlike) vector. The curve $\alpha$ is called spacelike (resp. timelike, lightlike) if it is for any $t \in I$ [8].

A non-lightlike curve $\alpha: I \subset R \longrightarrow E_{1}{ }^{3}$ is said to be parametrized by the pseudo arclength parameter s, if $\left|\left\langle\alpha^{\prime}(s), \alpha^{\prime}(s)\right\rangle\right|=1$. In this case, we call $\alpha$ is a unit speed curve.

For a unit speed non-lightlike curve $\alpha$ with a spacelike or timelike normal vector $N(s)$, the Frenet formulae are given in [8]. It's easy to calculate the formulae for arbitrary speed non-lightlike curves as follows.

If $\alpha$ is a timelike curve,

$$
\left[\begin{array}{l}
T^{\prime} \\
N^{\prime} \\
B^{\prime}
\end{array}\right]=\left[\begin{array}{ccc}
0 & \kappa v & 0 \\
\kappa v & 0 & \tau v \\
0 & -\tau v & 0
\end{array}\right]\left[\begin{array}{l}
T \\
N \\
B
\end{array}\right]
$$

If $\alpha$ is a spacelike curve with a spacelike normal vector $N(t)$,

$$
\left[\begin{array}{c}
T^{\prime} \\
N^{\prime} \\
B^{\prime}
\end{array}\right]=\left[\begin{array}{ccc}
0 & \kappa v & 0 \\
-\kappa v & 0 & \tau \nu \\
0 & \tau \nu & 0
\end{array}\right]\left[\begin{array}{l}
T \\
N \\
B
\end{array}\right]
$$

If $\alpha$ is a spacelike curve with a timelike normal vector $N(t)$,

$$
\left[\begin{array}{c}
T^{\prime} \\
N^{\prime} \\
B^{\prime}
\end{array}\right]=\left[\begin{array}{ccc}
0 & \kappa v & 0 \\
\kappa v & 0 & \tau \nu \\
0 & \tau v & 0
\end{array}\right]\left[\begin{array}{l}
T \\
N \\
B
\end{array}\right]
$$

where

$$
\kappa=\frac{\left\|\alpha^{\prime} \wedge \alpha^{\prime \prime}\right\|}{\left\|\alpha^{\prime}\right\|^{3}}, \tau=\frac{\operatorname{det}\left(\alpha^{\prime}, \alpha^{\prime \prime}, \alpha^{\prime \prime \prime}\right)}{\left\|\alpha^{\prime} \wedge \alpha^{\prime \prime}\right\|^{2}}, v=\sqrt{\left|\left\langle\alpha^{\prime}, \alpha^{\prime}\right\rangle\right|} .
$$

In the formulae above, we denote unit tangent vector with $T(t)$, unit binormal vector with $B(t)$, unit normal vector with $N(t)$. A regular timelike or spacelike curve $\alpha$ is a helix, if $\tau / \kappa$ is a constant function.

For a unit speed curve $\alpha$ in $R_{1}^{3}$, slant helix characterization is given in [1]. Also, some characterizations of Lorentzian unit speed curves which lies on $H^{2}$ or $S_{1}^{2}$ were investigated in $[9,10,11,12]$. With the help of these papers, we easily have the Lemmas for arbitrary speed curves below.

Lemma 2.1. Let $\alpha$ be a timelike curve in $R_{1}^{3}$. Then, $\alpha$ is a slant helix if and only if either one of the next two functions

$$
\frac{\kappa^{2}}{v\left(\tau^{2}-\kappa^{2}\right)^{3 / 2}}\left(\frac{\tau}{\kappa}\right)^{\prime} \quad \text { or } \frac{\kappa^{2}}{v\left(\kappa^{2}-\tau^{2}\right)^{3 / 2}}\left(\frac{\tau}{\kappa}\right)^{\prime}
$$

is constant everywhere $\tau^{2}-\kappa^{2}$ does not vanish.

Lemma 2.2. Let $\alpha$ be a spacelike curve in $R_{1}^{3}$ with a spacelike normal vector. Then, $\alpha$ is a slant helix if and only if either one of the next two functions

$$
\frac{\kappa^{2}}{v\left(\tau^{2}-\kappa^{2}\right)^{3 / 2}}\left(\frac{\tau}{\kappa}\right)^{\prime} \quad \text { or } \frac{\kappa^{2}}{v\left(\kappa^{2}-\tau^{2}\right)^{3 / 2}}\left(\frac{\tau}{\kappa}\right)^{\prime}
$$

is constant everywhere $\tau^{2}-\kappa^{2}$ does not vanish. 
Lemma 2.3. Let $\alpha$ be a spacelike curve in $R_{1}^{3}$ with a timelike normal vector. Then, $\alpha$ is a slant helix if and only if the function

$$
\frac{\kappa^{2}}{v\left(\tau^{2}+\kappa^{2}\right)^{3 / 2}}\left(\frac{\tau}{\kappa}\right)^{\prime}
$$

is constant.

Lemma 2.4. Let $\alpha$ be a spacelike curve in $R_{1}^{3}$ with a spacelike normal vector. Image of $\alpha$ lies on the pseudosphere (resp. hyperbolic plane) of radius $r$ and center $q$ if and only if

$$
\frac{1}{\kappa^{2}}-\left(\frac{1}{v \tau}\left(\frac{1}{\kappa}\right)^{\prime}\right)^{2}= \pm r^{2}(\text { resp. })
$$

where $r>0 \in R, \kappa \neq 0, \tau \neq 0$.

Lemma 2.5. Let $\alpha$ be a timelike curve in $R_{1}^{3}$. Image of $\alpha$ lies on the pseudosphere of radius $r$ and center $q$ if and only if

$$
\frac{1}{\kappa^{2}}+\left(\frac{1}{v \tau}\left(\frac{1}{\kappa}\right)^{\prime}\right)^{2}=r^{2}
$$

where $r>0 \in R, \kappa \neq 0, \tau \neq 0$.

Lemma 2.6. Let $\alpha$ be a spacelike curve in $R_{1}^{3}$ with a timelike normal vector. Image of $\alpha$ lies on the hyperbolic plane of radius $r$ and center $q$ if and only if

$$
\frac{-1}{\kappa^{2}}+\left(\frac{1}{v \tau}\left(\frac{1}{\kappa}\right)^{\prime}\right)^{2}=-r^{2}
$$

where $r>0 \in R, \kappa \neq 0, \tau \neq 0$.

Let $\gamma$ be a non-lightlike unit speed spherical curve with the arc-length parameter $s$ and denote $\gamma^{\prime}=t$ where $\gamma^{\prime}=d \gamma / d s$. If we set a vector $p=\gamma \wedge t$, by definition we have an orthonormal frame $\{\gamma, t, p\}$. This frame is called the pseudo-Sabban frame of $\gamma[5,6]$. Thus, we have the following Lemma .

Lemma 2.7. Let $\gamma(s)$ be a unit speed spherical curve in $R_{1}^{3}$, then (i) If $\gamma$ is a timelike curve on $S_{1}^{2}$ then,

$$
\begin{gathered}
\gamma^{\prime}=t \\
t^{\prime}=k_{g} p+\gamma \\
p^{\prime}=k_{g} t
\end{gathered}
$$

(ii) If $\gamma$ is a spacelike curve on $S_{1}^{2}$, then

$$
\begin{gathered}
\gamma^{\prime}=t \\
t^{\prime}=-k_{g} p-\gamma \\
p^{\prime}=-k_{g} t
\end{gathered}
$$

(iii) If $\gamma$ is a spacelike curve on $H^{2}$, then

$$
\begin{gathered}
\gamma^{\prime}=t \\
t^{\prime}=k_{g} p+\gamma \\
p^{\prime}=-k_{g} t
\end{gathered}
$$

where $k_{g}=\operatorname{det}\left(\gamma, t, t^{\prime}\right)$ the geodesic curvature of curve $\gamma$.

\section{Spherical helices on $S_{1}^{2}(r ; p)$ and $H^{2}(r ; p)$}

Let us take the curve

$$
\alpha(s)=b \int e^{\int k(s) d s} \gamma(s) d s+a
$$

at [4]. If we make the neccessary calculations, we have

$$
\begin{gathered}
\alpha^{\prime}(s)=b e^{\int k(s) d s} \gamma(s), \\
\alpha^{\prime \prime}(s)=b e^{\int k(s) d s}\left(k(s) \gamma(s)+\gamma^{\prime}(s)\right), \\
\alpha^{\prime \prime \prime}(s)=b e^{\int k(s) d s}\left(\left(k^{2}(s)+k^{\prime}(s)\right) \gamma(s)+2 k(s) \gamma^{\prime}(s)+\gamma^{\prime \prime}(s)\right) .
\end{gathered}
$$

If we calculate $\kappa, \tau$, and $v$ of the curve $\alpha$ by using the equations at (2.4) and (3.2), we find

$$
\begin{gathered}
\kappa(s)=\frac{1}{b e^{\int k(s) d s},} \\
\tau(s)=\frac{k_{g}(s)}{b e^{\int k(s) d s},} \\
v(s)=b e^{\int k(s) d s} .
\end{gathered}
$$


It's easy to see

$$
\begin{gathered}
\left\langle\alpha^{\prime}(s), \alpha^{\prime}(s)\right\rangle=b^{2} e^{2 \int k(s) d s}\langle\gamma(s), \gamma(s)\rangle, \\
T(s)=\gamma(s), \\
T^{\prime}(s)=t(s) .
\end{gathered}
$$

So, we can say if $\gamma$ is a unit speed spacelike curve which lies on $S_{1}^{2}$, then $\alpha$ is a spacelike curve with a spacelike normal vector $N$. If $\gamma$ is a unit speed spacelike curve which lies on $H^{2}$, then $\alpha$ is a timelike curve with a spacelike normal vector $N$. If $\gamma$ is a unit speed timelike curve which lies on $S_{1}^{2}$ then $\alpha$ is a spacelike curve with a timelike normal vector $N$. Now, we want to show, under which circumstances the curve $\alpha$ at equation (3.1) is a spherical helix on $S_{1}^{2}(r ; p)$.

Theorem 3.1. If the curve $\gamma$ is a unit speed spacelike curve with a constant geodesic curvature, which lies on $S_{1}^{2}$, the curve $\alpha$ defined by (3.1) is a spherical helix which lies on the pseudosphere of the radius $|b d|$ and of the center origin if and only if the function $k(s)=k_{g} \tanh \left[\left(k_{g}\right)(s-c)\right]$ where $b, c, d \in R$.

Proof. From (3.2), (3.3), and (3.4), we know the curve

$$
\alpha(s)=b \int e^{\int k(s) d s} \gamma(s) d s+a
$$

is a spacelike curve with a spacelike normal vector $N(s)$. So we need to use (2.8). Let's take the derivate of (2.8) with respect to $s$. Then, we have

$$
\left(\frac{1}{v}\left[\frac{1}{v \tau}\left(\frac{1}{\kappa}\right)^{\prime}\right]^{\prime}-\frac{\tau}{\kappa}\right)(s)=0
$$

By putting (3.3) in this equation, we have

$$
\begin{gathered}
\left(\frac{1}{b e^{\int k d s}}\left[\frac{1}{k_{g}}\left(b e^{\int k d s}\right)^{\prime}\right]^{\prime}-k_{g}\right)(s)=0 \\
k^{\prime}(s)+k^{2}(s)=k_{g}{ }^{2} .
\end{gathered}
$$

If we solve this differential equation, we have

$$
k(s)=k_{g} \tanh \left[\left(k_{g}\right)(s-c)\right]
$$

Conversely, if we take $k(s)=k_{g} \tanh \left[\left(k_{g}\right)(s-c)\right]$ in (14), then

$$
\int k(s) d s=\int k_{g} \tanh \left[\left(k_{g}\right)(s-c)\right] d s .
$$

Let $u=k_{g}(s-c)=k_{g} s-k_{g} c$ then $k_{g} d s=d u$, by using these equations

$$
\begin{aligned}
\int k(s) d s & =\int \tanh u d u \\
& =\ln \cosh u+\ln d \\
& =\ln \left[d \cosh \left(k_{g}(s-c)\right)\right]
\end{aligned}
$$

we have

$$
\begin{aligned}
\alpha(s) & =b \int e^{\int k(s) d s} \gamma(s) d s+a \\
& =b \int e^{\ln \left[d \cosh \left(k_{g}(s-c)\right)\right]} \gamma(s) d s+a \\
& =b \int d \cosh \left(k_{g}(s-c)\right) \gamma(s) d s+a
\end{aligned}
$$

where $c, d \in R$.

Now, we must show that curve $\alpha$ is spherical. If we use (2.8) to do it, we have

$$
\begin{aligned}
& \left.r^{2}=\left(\left(\frac{1}{\kappa^{2}}-\left(\frac{1}{v \tau}\left(\frac{1}{\kappa}\right)\right)^{\prime}\right)\right)^{2}\right)(s) \\
& =\left(b^{2} e^{2 \int k d s}\left(1-\frac{k^{2}}{k_{g}^{2}}\right)\right)(s) \\
& =b^{2} d^{2} \cosh ^{2}\left(k_{g}(s-c)\right)\left(\frac{1}{\cosh ^{2}\left(k_{g}(s-c)\right)}\right) \\
& =b^{2} d^{2}
\end{aligned}
$$

Therefore, it can be said that the curve $\alpha$ lies on $S_{1}^{2}$ which has a radius $|b d|$. 
Now, we can give another theorem.

Theorem 3.2. If the curve $\gamma$ is a unit speed spacelike curve with a constant geodesic curvature, which lies on $H^{2}$, the curve $\alpha$ defined by (3.1) is a spherical helix which lies on the pseudosphere of the radius $|b d|$ and of the center origin if and only if the function $k(s)=k_{g} \tan \left[\left(k_{g}\right)(s-c)\right]$ where $b, c, d \in R$.

Proof. By using (2.9) instead of (2.8) in Theorem 3.1, the proof is similar.

Theorem 3.3. If the curve $\gamma$ is a unit speed timelike curve with a constant geodesic curvature, which lies on $S_{1}^{2}$, the curve $\alpha$ defined by (3.1) is a spherical helix which lies on the hyperbolic plane of the radius $|b d|$ and of the center origin if and only if the function $k(s)=k_{g} \tanh \left[\left(k_{g}\right)(s-c)\right]$ where $b, c, d \in R$.

Proof. By using (2.10) instead of (2.8) in Theorem 3.1, the proof is similar.

Example 3.4. Let's take $\gamma(s)=\{\sqrt{2} \cos (s / \sqrt{2}), \sqrt{2} \sin (s / \sqrt{2}), 1\}$, we know that $\gamma$ is a spacelike curve on $S_{1}^{2}$ with the geodesic curvature $\sqrt{2}$. Then due to Theorem 3.1,

$$
k(s)=k_{g} \tanh \left[\left(k_{g}\right)(s-c)\right]
$$

and

$$
\alpha(s)=b \int d \cosh \left(k_{g}(s-c)\right) \gamma(s) d s+a
$$

where $b, c, d \in R$. If we take $b=2, c=0, d=1$; then, we have

$$
\begin{gathered}
\alpha_{1}(s)=2 \cosh (s / \sqrt{2}) \sin (s / \sqrt{2})+2 \cos (s / \sqrt{2}) \sinh (s / \sqrt{2}) \\
\alpha_{2}(s)=-2 \cos (s / \sqrt{2}) \cosh (s / \sqrt{2})-2 \sin (s / \sqrt{2}) \sinh (s / \sqrt{2}) \\
\alpha_{3}(s)=2 \sqrt{2} \sinh (s / \sqrt{2})
\end{gathered}
$$

where $\alpha(s)=\left(\alpha_{1}(s), \alpha_{2}(s), \alpha_{3}(s)\right)$ and $a=(0,0,0)$

Example 3.5. Let's take $\gamma(s)=\{\cos (s), \sin (s), \sqrt{2}\}$, we know that $\gamma$ is a spacelike curve on $H^{2}$ with the geodesic curvature $\sqrt{2}$. Then, due to Theorem 3.2,

$$
k(s)=k_{g} \tan \left[\left(k_{g}\right)(s-c)\right]
$$

and

$$
\alpha(s)=b \int d \cos \left(k_{g}(s-c)\right) \gamma(s) d s+a
$$

where $b, c, d \in R$. If we take $b=2, c=0, d=1$; then, we have

$$
\begin{gathered}
\alpha_{1}(s)=-2 \cos (\sqrt{2} s) \sin (s)+2 \sqrt{2} \cos (s) \sin (\sqrt{2} s) \\
\alpha_{2}(s)=2 \cos (s) \cos (\sqrt{2} s)+2 \sqrt{2} \sin (s) \sin (\sqrt{2} s) \\
\alpha_{3}(s)=2 \sin (\sqrt{2} s)
\end{gathered}
$$

where $\alpha(s)=\left(\alpha_{1}(s), \alpha_{2}(s), \alpha_{3}(s)\right)$ and $a=(0,0,0)$

Example 3.6. Let's take $\gamma(s)=\left\{\frac{1}{\sqrt{3}} \cosh (\sqrt{3} s), \frac{\sqrt{2}}{\sqrt{3}}, \frac{1}{\sqrt{3}} \sinh (\sqrt{3} s)\right\}$, we know that $\gamma$ is a timelike curve on $S_{1}^{2}$ with the geodesic curvature $\sqrt{2}$. Then, due to Theorem 3.3,

$$
k(s)=k_{g} \tanh \left[\left(k_{g}\right)(s-c)\right]
$$

and

$$
\alpha(s)=b \int d \cosh \left(k_{g}(s-c)\right) \gamma(s) d s+a
$$

where $b, c, d \in R$. If we take $b=2, c=0, d=1$; then, we have

$$
\begin{gathered}
\alpha_{1}(s)=-2 \sqrt{\frac{2}{3}} \cosh (\sqrt{3} s) \sinh (\sqrt{2} s)+2 \cosh (\sqrt{2} s) \sinh (\sqrt{3} s) \\
\alpha_{2}(s)=\frac{2 \sinh (\sqrt{2} s)}{\sqrt{3}} \\
\alpha_{3}(s)=2 \cosh (\sqrt{2} s) \cosh (\sqrt{3} s)-2 \sqrt{\frac{2}{3}} \sinh (\sqrt{2} s) \sinh (\sqrt{3} s)
\end{gathered}
$$

where $\alpha(s)=\left(\alpha_{1}(s), \alpha_{2}(s), \alpha_{3}(s)\right)$ and $a=(0,0,0)$ 

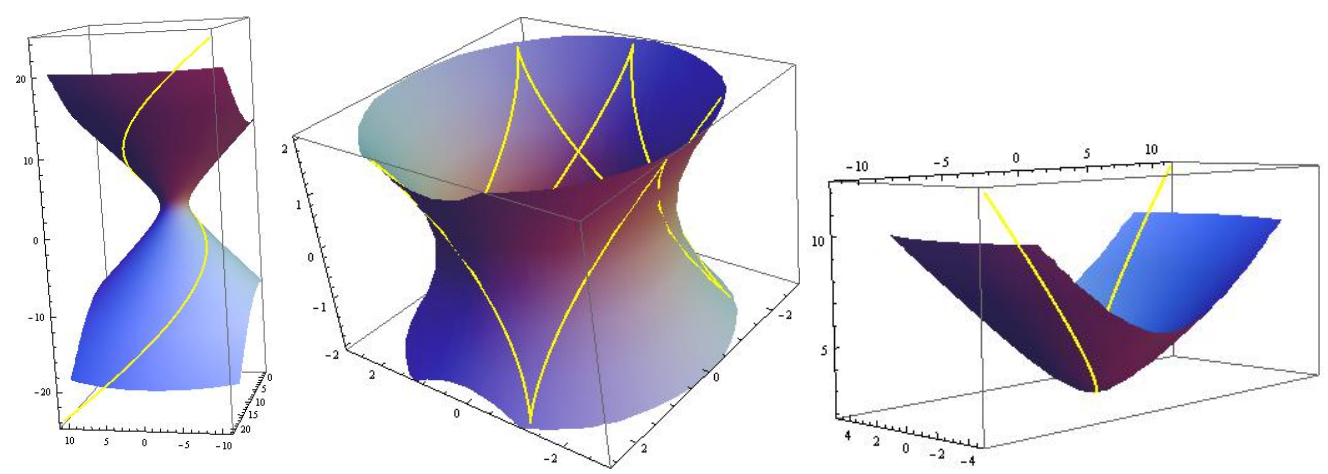

Figure 3.1: Spherical Helices (Resp. Example 1,2, and 3)

\section{Constructing slant helices from unit speed spherical curves}

In this section, we want to give some characterizations about slant helices.

Theorem 4.1. Let $\gamma(s)$ be a unit speed spacelike curve on $S_{1}^{2} ; b, m, n$ be constant numbers; and a be a constant vector. The geodesic curvature of $\gamma(s)$ satisfies

$$
k_{g}{ }^{2}(s)=\frac{(m s+n)^{2}}{1+(m s+n)^{2}}
$$

if and only if

$$
\alpha(s)=b \int e^{\int k(s) d s} \gamma(s) d s+a
$$

is a spacelike slant helix with a spacelike normal vector.

Proof. Let, for $\gamma$

$$
k_{g}^{2}(s)=\frac{(m s+n)^{2}}{1+(m s+n)^{2}} .
$$

From (3.2), (3.3), and (3.4), we know $\alpha$ is a spacelike curve with a spacelike normal vector $N$. So; from (2.6), the geodesic curvature of the spherical image of the principal normal indicatrix of $\alpha$ is as follows

$$
\begin{aligned}
\sigma(s) & =\left(\frac{\kappa^{2}}{v\left(\kappa^{2}-\tau^{2}\right)^{3 / 2}}\left(\frac{\tau}{\kappa}\right)^{\prime}\right)(s) \\
& =\left(\frac{\frac{1}{v^{2}}}{v\left(\frac{1}{v^{2}}-\frac{k_{g}^{2}}{v^{2}}\right)^{3 / 2}} k_{g}^{\prime}\right)(s) .
\end{aligned}
$$

So, we have

$$
\sigma(s)=\frac{k_{g}{ }^{\prime}(s)}{\left(1-k_{g}{ }^{2}(s)\right)^{3 / 2}}
$$

Now, let's take $u(s)=m s+n$, then we have (4.1)

$$
k_{g}^{2}(s)=\frac{u^{2}(s)}{1+u^{2}(s)} .
$$

If we take the derivates of the both sides of (4.3) with respect to $s$, we have

$$
\begin{aligned}
& 2 k_{g}(s) k_{g}^{\prime}(s)=\left(\frac{2 u u^{\prime}\left(1+u^{2}\right)-\left(2 u u^{\prime}\right) u^{2}}{\left(1+u^{2}\right)^{2}}\right)(s) \\
& k_{g}(s) k_{g}^{\prime}(s)=\left(\frac{u u^{\prime}}{\left(1+u^{2}\right)^{2}}\right)(s) \\
& k_{g}^{\prime}(s)=\left(\left(\frac{u u^{\prime}}{\left(1+u^{2}\right)^{2}}\right)\left(\varepsilon \sqrt{\frac{1+u^{2}}{u^{2}}}\right)\right)(s)
\end{aligned}
$$


where $\varepsilon= \pm 1$. Putting (4.3) and (4.4) in (4.3), we have

$$
\begin{aligned}
\sigma(s) & =\frac{k_{g}{ }^{\prime}(s)}{\left(1-k_{g}{ }^{2}(s)\right)^{3 / 2}} \\
& =\left(\varepsilon \frac{\sqrt{1+u^{2}} u u^{\prime}}{|u|\left(1+u^{2}\right)^{2}}\left(1+u^{2}\right)^{3 / 2}\right)(s) \\
& =\varepsilon \frac{m s+n}{|m s+n|} m \\
& =\varepsilon m
\end{aligned}
$$

which is constant.

Conversely, let $\alpha(s)$ be a spacelike slant helix, then the geodesic curvature of the spherical image of the principal normal indicatrix of $\alpha$ is a constant function. So, we can take

$$
\sigma(s)=\left(\frac{\kappa^{2}}{v\left(\kappa^{2}-\tau^{2}\right)^{3 / 2}}\left(\frac{\tau}{\kappa}\right)^{\prime}\right)(s)=m
$$

where $m \in R$. Therefore, from (4.2)

$$
\begin{aligned}
m & =\left(\frac{\kappa^{2}}{v\left(\kappa^{2}-\tau^{2}\right)^{3 / 2}}\left(\frac{\tau}{\kappa}\right)^{\prime}\right)(s) \\
& =\frac{k_{g}{ }^{\prime}(s)}{\left(1-k_{g}{ }^{2}(s)\right)^{3 / 2}}
\end{aligned}
$$

If we solve this differential equation, we have

$$
\frac{k_{g}(s)}{\sqrt{1-k_{g}^{2}(s)}}=m s+n
$$

where $n \in R$. Then,

$$
k_{g}^{2}(s)=\frac{(m s+n)^{2}}{1+(m s+n)^{2}} .
$$

Theorem 4.2. Let $\gamma(s)$ be a unit speed spacelike curve on $H^{2} ; b, m, n$ be constant numbers; and a be a constant vector. The geodesic curvature of $\gamma(s)$ satisfies

$$
k_{g}^{2}(s)=\frac{(m s+n)^{2}}{1+(m s+n)^{2}}
$$

if and only if

$$
\alpha(s)=b \int e^{\int k(s) d s} \gamma(s) d s+a
$$

is a timelike slant helix with a spacelike normal vector.

Proof. By using (2.5) instead of (2.6) in Theorem 4.1, the proof is similar.

Theorem 4.3. Let $\gamma(s)$ be a unit speed timelike curve on $S_{1}^{2} ; b, m, n$ be constant numbers; and a be a constant vector. The geodesic curvature of $\gamma(s)$ satisfies

$$
k_{g}^{2}(s)=\frac{(m s+n)^{2}}{1-(m s+n)^{2}}
$$

if and only if

$$
\alpha(s)=b \int e^{\int k(s) d s} \gamma(s) d s+a
$$

is a spacelike slant helix with a timelike normal vector.

Proof. By using (2.7) instead of (2.6) in Theorem 4.1, the proof is similar. 


\section{References}

[1] Ali Ahmad, T. and Lopez, R., Slant helices in Minkowski space $E_{1}^{3}$, J. Korean Math. Soc. 48, no. 1, 159-167. 2011.

[2] Altunkaya, B. and Kula, L., Some characterizations of slant and spherical helices due to sabban frame, Mathematical Sciences and Applications E-Notes, Vol 3, No. 2, 64-73, 2015

[3] Babaarslan, M. and Yayli, Y., On spacelike constant slope surfaces and Bertrand curves in Minkowski 3-space, Annals of the Alexandru Ioan Cuza University -Mathematics, 2015. doi:10.1515/aicu-2015-0009.

[4] Encheva, R. and Georgiev, G., Shapes of space curves, Journal for Geometry and Graphics, Vol 7, No. 2, 145-155, 2003.

[5] Güner, G. and Ekmekci, N., On the spherical curves and Bertrand curves in Minkowski 3-space, J. Math. Comput. Sci., No. 4, 898-906, 2012.

[6] Izuyama, S., Pei, D.H., Sano, T., and Torii, E., Evolutes of hyperbolic plane curves, Acta Mathematica Sinica, Vol.20, no.3, pp. 543-550, 2004.

[7] Izumiya, S. and Takeuchi, N., New special curves and developable surfaces, Turk. J. Math. 28, 153-163, 2004.

[8] Lopez, R., Differential Geometry of Curves and Surfaces in Lorentz-Minkowski space, arXiv:0810.3351v1 [math.DG], 2008.

[9] Pekmen, Ü. and Paşalı, S., Some characterizations of Lorentzian spherical spacelike curves, Mathematica Moravica 3, 31-37, 1999.

[10] Petrović-Torgašev, M. and S̆ućurović, E., Some characterizations of Lorentzian spherical spacelike curves with the timelike and the null principal normal, Math. Moravica, 4, 83-92, 2000.

[11] Petrović-Torgašev, M. and Šćurović, E., Some characterizations of Lorentzian spherical timelike and null curves, Matematički Vesnik, 53, 21-27, 2001.

[12] Petrović-Torgašev, M. and Sućurović, E., Some characterizations of the spacelike, the timelike and the on the pseudohyperbolic space $H_{0}^{2}$ in $E_{1}^{3}$, Krugajevac J. Math., 22, 71-82, 2000. 